\title{
Intravenous Leiomyomatosis of the Uterus: Case Report
}

\section{Jellouli MA* and Mathis J}

Department of Gynecology and Obstetrics, Biel Spitalzentrum, Switzerland

*Corresponding Author: Jellouli MA, Department of Gynecology and Obstetrics, Biel Spitalzentrum, Switzerland.
Received: December 14, 2021

Published: January 10, 2022

(C) All rights are reserved by Jellouli MA and

Mathis J.

\section{Abstract}

Intravenous leiomyomatosis (IVL) is a benign and rare pathology, characterized by intravenous proliferation of a histologically benign smooth muscle cell. It can result in systematic complications, especially cardiac, with threatening outcomes if remained untreated.

Intravenous leiomyomatosis (IVL) is probably underestimated, due to a likely easily missed diagnosis.

Introduction: We present a case of an intravenous leiomyomatosis without extra-pelvic involvement, with a brief review of this pathology.

Case Report: A total hysterectomy and bilateral oophorectomy was planned in a 50-year-old woman was because of recurrent pelvic pain with uterus myxomatosis detected in ultrasound. During the surgery, intravenous leiomyomatosis diagnosis was not suspected. Pathological analysis confirmed the unexpected diagnosis. Later, other imaging exams were performed without detecting any extrapelvic localization. The patient remained with no evidence of disease after 2 years of follow-up.

Conclusion: Although Intravenous leiomyomatosis is rare, it can cause serious complications. Early diagnosis followed by an appropriate treatment (Hysterectomy and oophorectomy or anti-estrogens therapy) are necessary to patient outcome. Further exams and long-term follow-up is needed to exclude systemic metastasis or recurrences.

Keywords: Intravenous Leiomyomatosis; Myomas; Uterus

\section{Introduction}

Intravenous leiomyomatosis (IVL) is an uncommon and a severe disease in women between 20 - and 70-years old age.

IVL was firstly described by Birch-Hirschfeld in 1896 [1], and Dürck [2] reported the first case of extension of IVL to the heart in 1907.

This enlarged smooth muscle tumor is benign, with nonspecific clinical presentation, thus many IVL patients are misdiagnosed or only diagnosed after the tumor has reached the heart and sometimes cause sudden death by obstruction of the total outflow tract.

\section{Case Report}

A 50-year-old woman, Gravida 3, Para 2, with a personal history of 2 cesarean sections, and family history of endometrial cancer by her older sister. The contraception method is hormonal IUD. The patient was complaining of regular pelvic pain, with recurrent pollakiuria. Her family doctor referred her to our hospital for a gynecological consultation.

The gynecological examination revealed a large uterus reaching the umbilicus without any other palpable masses.

The transvaginal ultrasound showed an antevert uterus, $130 *$ $90 * 80 \mathrm{~mm}$, with 3 myomas, type FIGO 4 of respectively $45^{*} 65 \mathrm{~mm}$, $58 * 47 \mathrm{~mm}$ et $56^{*} 60 \mathrm{~mm}$. The Adnexa were free and endometrium thin (IUD in right place).

The patient was submitted to a total hysterectomy with bilateral oophorectomy by laparotomy (Pfannenstiel). 
The surgery went well and the findings included a large uterus, with 3 intra-mural fibroids; the ovaries and fallopian tubes were macroscopically normal within parametrial vessels l (Figure 1).

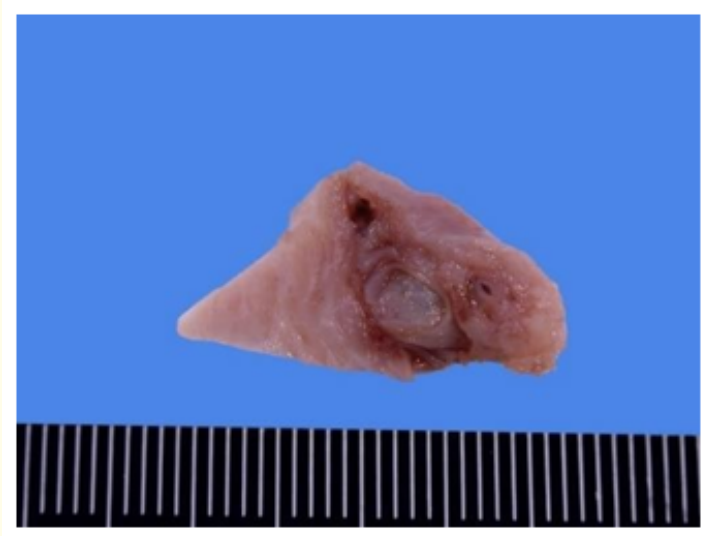

Figure 1: Worm-like plugs.

No post-operative complication was noticed.

The anatomopathological analysis concluded to a discrete chronic cervicitis, atrophic endometrium and multiple intramural leiomyomas with intravenous leiomyomatosis (IVL) (Figure 2).

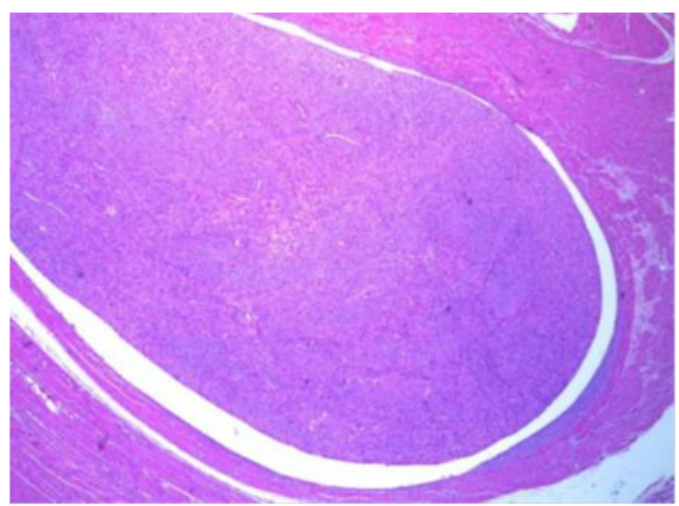

Figure 2: Microscopic findings.

After discussion in tumor-board, and due to the risk of venous and cardiac localizations, a cardiac Ultrasound exam and a thoracoabdominal CT scan were indicated. Both did not find any secondary localization of intravenous leiomyomatosis (IVL).
During 2 years of survey, the patient was controlled every 6 months with no plains and normal gynecological exam. Cardiac Ultrasound and thoraco- Abdominal CT scan were repeated every year with no special findings.

\section{Discussion}

Intravenous leiomyomatosis is a rare entity characterized by intravascular nodular masses of histologically benign smooth muscle cells growing in uterine and pelvic veins. Between 400 to 450 cases are already reported in the literature and less than 100 cases with cardiac [3-4,6]. LIV reaches patients from 20 and 70 years of age, with an average age of 45 years [5-6]. Pre-menopausal and multiparous females are most preferentially reached [7].

Extra-uterine involvement is seen in $30 \%$ to $80 \%$ of cases of uterine intravenous leiomyomatosis, with cardiac involvement in $10 \%$ to $30 \%$ [8-9].

The etiology of LIV is still uncertain, but two hypotheses are accepted

- Invasion of the vessels by a uterine leiomyoma $[7,10]$ through hematogenous dissemination

- Direct metaplasia from the venous endothelium $[7,10]$.

However, the recent study of Ma [11] from Peking Hospital, the second theory is questioned. In fact, among 76 patients with IVL in this retrospective study of 14 years, immunohistochemical staining indicated Desmin and SMA-positive cells, confirming the origin of tumor thrombus from smooth muscle cell. On the opposite, CD10, CD31, or CD34 staining was negative, leading to a conclusion that the tumor origin is not from endometrial stroma cell or vascular endothelium cell.

In 2018, Wolf., et al. has published a new entity, called "peri-vascular leiomyomatosis" (PVL) [12], that could be the first stage of LIV, as a form of leiomyomatosis parasitic. They described an atypical localization (adenitises and media of the saphen vein) without intravascular localization at first, without excluding an evolution to LIV. This finding could be at the origin of a new etiological hypothesis of the LIV.

In our case, IVL was not suspected before surgery, clinical signs of IVL, when localized in the pelvis are non-specific, and similar to those of uterus myxomatosis, such as pelvic pain, abnormal vaginal 
bleeding urinary signs [6]. Clinical examination usually reveals an enlarged uterus or a pelvic mass. The diagnosis is then often made during the analysis of the hysterectomy piece.

LIV can spread in the venous circulation, most often starting from the uterine vein and rarely from the ovarian vein [13].

When extension starts in the uterine vein, it can reach the internal and common iliac veins and then in the vena cava inferior (VCI). But when extension starts from the ovarian vein, it can directly attend the VCI on the right and via the renal vein on the left, avoiding the iliac veins.

Starting from VCI, when it's not treated, extension can reach the right atrium and pulmonary arteries. There are also forms associated with pulmonary nodules or with total cardiac damage.

Extra-pelvic vessels attempt (especially VCI), is associated with venous return anomalies, with congestive right heart failure signs: chest pain, dyspnea, oedema of lower limbs, syncope and pulmonary embolism. In case of cardiac involvement with obstruction of the tricuspid valve, the risk is cardiac arrest and sudden death $[14,15]$.

Cardiac ultrasound can be used to suggest the diagnosis of LIV with cardiac damage. The most frequent situation is an adult woman with an uterus myxomatosis, and right atrium mass taking origin from the vena cava and without argument for invasion endothelial or endocardium (mobile mass in the VCI and in the endocardium [13]. The tumor takes on the appearance of a mobile and free serpentine mass in the vessels and in the heart [10], without invasion of the endothelium nor the endocardium. The main differential diagnosis is the cardiac myxoma which is specifically intracardiac (usually in the left atrium), without a portion in the IVC, and adhere to the endocardium, especially at the level of the inter septum auricular [10]. Other possible differential diagnoses are Cardiac metastases (solid mass fixed to the wall), a thrombus (postoperative context with venous stasis) or a cardiac leiomyosarcoma (solid mass attached to the wall) [13].

The same signs are founded in CT-scanner and magnetic resonance imaging (MRI) he same appearance $[13,16]$.

Ma., et al. divided the IVL localization in to 4 Stages, starting from uterus (Stage one) to heart and pulmonary involvements (Stage 4) [11] (Figure 3).

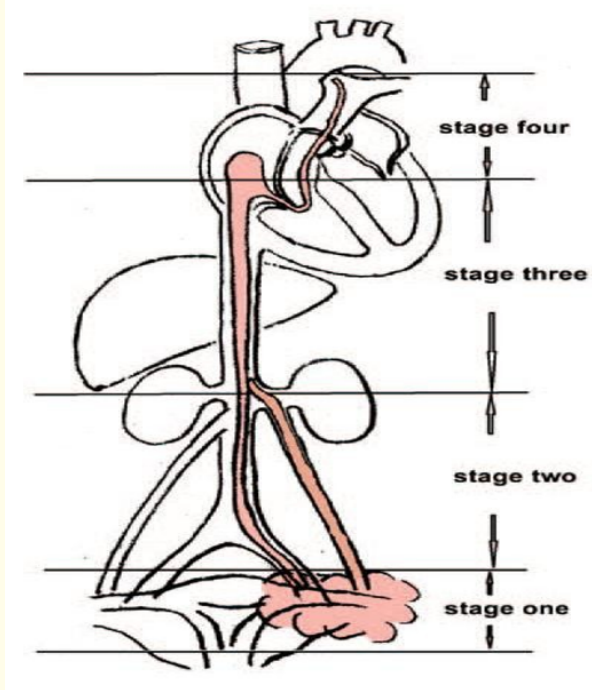

Figure 3: Stage and origin of intravenous leiomyomatosis according to Ma et all.

- $\quad$ Stage 1: Invasion of the uterine vessels without extra pelvic invasion.

- $\quad$ Stage 2: Extension into the abdominal cavity without damage to the vein renal.

- $\quad$ Stage 3: The renal vein and VCI as well as the atrium right are invaded.

- $\quad$ Stage 4: Invasion of the pulmonary arteries and/or the presence of pulmonary metastases [13].

According to the study [11], of 76 patients who had been treated for LIV, 41 patients (53.9\%) were grade 2 or higher. In the literature, cardiac involvement is estimated for approximately $10 \%$ $40 \%$ of LIV cases $[13,17,18]$.

Our case is classified as a stage 1 .

Concerning treatment, it's based primary on radical surgery, including a total hysterectomy with adnexectomy, associated with complete resection of intravenous tumors and intracardiac $[3,7,10]$.

Infact, the risk of recurrence is directly correlated to the lack of complete resection $[10,11]$. In the study of Ma and all, the 4 cases of recurrence have occurred in stage 1 patients who refused to be treated by hysterectomy and adnexectomy [11]. 
If we consider the fact that estrogen and progesterone receptors are present in the leiomyoma cells, bilateral salpingoophorectomy is necessary, and exogenous estrogens must be avoided. For the non-castration cases, post-surgery hormone therapy can also be considered. Gonadotropin-releasing hormone agonists or antiestrogen therapy have also been used to prevent tumor growth and to reduce the tumor.

In case of stage 1 , surgery is simple and based on a total hysterectomy with bilateral adnexectomy. For the other stages, a multidisciplinary team (gynecologists, vascular surgeons, anesthetists) must be involved in the management of the various extra-uterine tumors. Extracorporeal circulation should be used in stage 3 and $4[11,13]$.

The vascular extension, often described as a worm floating in the venous cavity with adhesion to the lower pelvic area, was the basis for the tumour stripping successfully carried out by the Harris team by approaching this adherent area [20]. In case of cardiac involvement, a sternotomy with a direct cardiac approach under extracorporeal circulation is necessary with tumor stripping via a cardiac and then abdominal approach in two stages [13]. The only alternative to surgery in this case is to perform a sternotomy with a direct cardiac approach under extracorporeal circulation [20].

The only alternative to surgery if complete resection is not possible or if the patient refuses is chemical castration. Gonadotropin releasing hormone (GnRH) analogues (a-GnRH), selective estrogen receptor modulators (SERMs), and antiromantics agents (AAs) $[21,22]$ can be used to treat the disease. It can also be used to prevent recurrence in cases of incomplete resection for 6 months, according to Ma [11].

Recurrence is main risk of LIV. The rate varies from $22.2 \%$ [3] to $30 \%[10,17,18]$. In the case of complete resection, the rate decreases to $7.6 \%[11]$.

Risk factors are young patients age, initial tumor size, incomplete resection without postoperative hormone therapy, uterine and ovarian preservation $[3,11]$.

The addition of chemical castration in case of partial resection would decrease the risk of recurrence. Recurrence may occur several years after surgery and may consist of venous or abdominal recurrence with multiple pelvic masses $[7,18]$. In case of recurrence, further radical surgery with complete resection significantly improves the prognosis [7].

Long-term monitoring is recommended. It is based on CT or MRI $[7,18]$ between 3 and 6 months $[7,17,18]$ after surgery and then regularly every 2 to 5 years, depending on the grade and completeness of the resection.

In our case, the patient remained with no evidence of disease after 2 years of follow-up (US and CT).

\section{Conclusion}

IVL is a rare disease. The onset is insidious and difficult to diagnose in the early stage. However, IVL is currently most often treated surgically and with antiestrogen therapy to suppress estrogen. Complete removal of the tumor is crucial to avoid recurrence of IVL. Incomplete or delayed resection can lead to serious complications such as recurrence or death. Long-term survey of minimum 5 years is necessary to avoid recurrence.

\section{Bibliography}

1. Birch-Hirschfeld FV., et al. "Royal College of Physicians of Edinburgh". Lehrbuch der Pathologischen Anatomie (1887): 226258.

2. Dürck H. "Ueber ein kontinvierlich durch die learned Holvene in das Herz vorwachsendes Fibromyom des Uterus". Münchener Medizinische Wochenschrift 54 (1907): 1154.

3. Low H-Y., et al. "Intravenous leiomyomatosis of the uterus: a clinicopathological analysis of nine cases and literature review". Taiwanese Journal of Obstetrics and Gynecology 56 (2017): 362-365.

4. E Declas and JP Lucot. "Extra uterine leiomyomatosis: Review of the literature”. Gynécologie Obstétrique Fertilité and Sénologie 47 (2019): 582-590.

5. Kim Y-N., et al. "Aberrant uterine leiomyomas with extrauterine manifestation: intravenous leiomyomatosis and benign metastasizing leiomyomas". Obstetrics and Gynecology Science 61 (2018): 509-519.

6. Qingbo Su., et al. "Intravenous Leiomyomatosis of the Uterus: A Retrospective Single-Center Study in 14 Cases". Hindawi BioMed Research International (2020). 
7. Valdés Devesa V., et al. "Update on intravenous leiomyomatosis: report of five patients and literature review". European Journal of Obstetrics and Gynecology and Reproductive Biology 171 (2013): 209-213.

8. Fornaris RJ., et al. "Multimodality Evaluation of Intravenous Leiomyomatosis: A Rare, Benign but Potentially Life- Threatening Tumor". American Journal of Case Reports 16 (2015): 794-800.

9. Nakai G., et al. "Uterine Intravenous Leiomyomatosis with Cardiac Extension: Radiologic Assessment withSurgical and Pathologic Correlation". Case Reports in Obstetrics and Gynecology (2015): 2015576743.

10. Vaquero ME., et al. "Uterine smooth-muscle tumors with unusual growth patterns". The Journal of Minimally Invasive Gynecology 16 (2009): 263-268.

11. Ma G., et al. "Different surgical strategies of patients with intravenous leiomyomatosis". Medicine 95 (2016): 37.

12. Wolfe S., et al. "Perivascular leiomyomatosis: a unique case report”. International Journal of Surgical Pathology 26.7 (2018): 676-679.

13. Li R., et al. "Intravenous leiomyomatosis with intracardiac extension: echocardiographic study and literature review". Texas Heart Institute Journal 41 (2014): 502-506.

14. Clay TD., et al. "Intravenous leiomyomatosis with intracardiac extension-a review of diagnosis and management with an illustrative case". Surgical Oncology 22 (2013): e44-52.

15. Gu X., et al. "Intracardiac leiomyomatosis: clinical findings and detailed echocardiographic features-a Chinese institutional experience". The Journal of the American Society of Echocardiography 27 (2014): 1011-1016.

16. Meddeb M., et al. "The heart as a site of metastasis of benign metastasizing leiomyoma: case report and review of the literature". Case Reports in Cardiology 2018 (2018): 7231326.

17. Fasih N., et al. "Leiomyomas beyond the uterus: unusual locations, rare manifestations". Radiographics 28 (2008): 19311948.

18. Mahmoud MS., et al. "Leiomyomas beyond the uterus; benign metastasizing leiomyomatosis with paraaortic metastasizing endometriosis and intravenous leiomyomatosis: a case a case series and review of the literature". Archives of Gynecology and Obstetrics 291 (2015): 223-230.
19. Lin J., et al. "Pelvic intravascular leiomyomatosis associated with benign pulmonary metastasizing leiomyoma: clinicopathologic, clonality, and copy number variance analysis". International Journal of Gynecological Pathology 33 (2014): 140-145.

20. Harris LM and Karakousis CP. "Intravenous leiomyomatosis with cardiac extension tumor thrombectomy through an abdominal approach". Journal of Vascular Surgery 31 (2000): 1046-1051.

21. Mizoguchi C., et al. "Intravenous leiomyomatosis treated with radical hysterectomy and adjuvant aromatase inhibitor therapy". Journal of Obstetrics and Gynaecology Research 42 (2016): 1405-1408.

22. Biri A., et al. "Intravenous leiomyomatosis treated with aromatase inhibitor therapy". International Journal of Gynecology and Obstetrics 101 (2008): 299-300.

\section{Assets from publication with us}

- Prompt Acknowledgement after receiving the article

- Thorough Double blinded peer review

- Rapid Publication

- Issue of Publication Certificate

- High visibility of your Published work

Website: www.actascientific.com/

Submit Article: www.actascientific.com/submission.php

Email us: editor@actascientific.com

Contact us: +919182824667 